IEEE.org IEEE Xplore Digital Library IEEE-SA IEEE Spectrum More Sites

Conferences > 2018 IEEE International Sympo...

\title{
Earth-Space Path Cloud Attenuation Distribution Study
}

5

Author(s)

O. M. Adewusi ; T.V Omotosho ; M. L. Akinyemi ; S. A. Akinwumi ; O. O. Ometan

View All Authors

Abstract

Document Sections

I.

Introduction

II.

Data Source

III.

Result and Discussion

IV.

Conclusion

Abstract:

The study investigates the impact of cloud attenuation at C, Ku, Ka, and V-bands in West Africa. Cloud attenuation statistics deducted from extracted data obtained from ITU-R study group data bank for the seventeen countries in the region, were used to determine the average satellite link margin for the region. Analysis of their cloud attenuation contour maps shows the average cloud attenuation for uplink and downlink: at the c-band are $0.17 \mathrm{~dB}$ and $0.095 \mathrm{~dB}$; at Ku-band are $0.875 \mathrm{~dB}$ and $0.665 \mathrm{~dB}$; at Ka-band $3.75 \mathrm{~dB}$ and $1.775 \mathrm{~dB}$; and at $\mathrm{V}$-band are $9.25 \mathrm{~dB}$ and $6.6 \mathrm{~dB}$ respectively in the tropical wet/dry climatic part of the region, while those for the tropical wet and tropical semi-arid climatic parts of the region are relatively higher and substantially lower respectively. Hence a margin of $1.8 \mathrm{~dB}$ and $3.8 \mathrm{~dB} ; 6.3 \mathrm{~dB}$ and 9 $\mathrm{dB}$ for cloud attenuation in the uplink and downlink at Ka and $\mathrm{V}$-band respectively are recommended.

Published in: 2018 IEEE International Symposium on Antennas and Propagation \& USNC/URSI National Radio Science Meeting 
Date of Conference: 8-13 July 2018

Date Added to IEEE Xplore: 14 January 2019

INSPEC Accession Number: 18376812

DOI: 10.1109/APUSNCURSINRSM.2018.8608306

Publisher: IEEE

Conference Location: Boston, MA, USA

I. Introduction

The effects of cloud constituents - suspended water droplets (SWD) and suspended ice crystals (SIC) are consistent and dynamic problem [1] in the satellite communication industry that continually increase frequencies of operation in response to market bandwidth demand, while the increases in transmission frequency are phenomenally accompanied by directly proportional hydrometeors attenuation [2].

Generally, cloud attenuation reduction of the amplitude of a propagating signal with respect to distance travelled along its transmission path is due to relaxation losses in the molecules of the SWD [3]. Signal transmission loss occur when propagating signal's energy is absorbed at high frequencies due to rotational and vibrational transitions in atmospheric polar molecules such as multitudes of tiny water droplets in clouds [4]. Previous research works have established that transmission frequencies above $3 \mathrm{GHz}$ have associated increase in signal propagation impairment in the troposphere [2]. Electromagnetic signals parameters include the signal amplitude, frequency, phase, bandwidth and polarization [2]; while the cloud properties affecting radio propagation also include cloud liquid water and cloud type's frequency of occurrence [5].

Sign in to Continue Reading

Authors

Simulation of the impact of cloud induced scintillations on satellite links

1993 Eighth International Conference on Antennas and Propagation

Published: 1993

Cloud attenuation on satellite links in the Ka/V-band and the effect of changes in the effective cloud temperature

2012 6th European Conference on Antennas and Propagation (EUCAP)

Published: 2012 
View More

Top Organizations with Patents on Technologies Mentioned in This Article

Advertisement

IEEE Account

Change Username/Password

Update Address

Need Help?

US \& Canada: +1 8006784333

Worldwide: +17329810060 